Review began 09/29/2021 Review ended 10/08/2021 Published 10/13/2021

๑) Copyright 2021

Aleem et al. This is an open access article distributed under the terms of the Creative Commons Attribution License CC-BY 4.0. which permits unrestricted use, distribution, and reproduction in any medium, provided the original author and source are credited.

\title{
Prevalence of Bacteria and Antimicrobial Resistance Genes in Hospital Water and Surfaces
}

Maira Aleem ${ }^{1}$, Abdul R. Azeem ${ }^{2}$, Sidra Rahmatullah ${ }^{3}$, Sufyan Vohra ${ }^{3}$, Shumyila Nasir ${ }^{3}$, Saadia Andleeb 3

1. Biotechnology, Combined Military Hospital $(\mathrm{CMH})$ - Lahore Medical College and Institute of Dentistry, Lahore, PAK 2. General Medicine, Combined Military Hospital (CMH), Lahore, PAK 3. Atta-ur-Rahman School of Applied Biosciences, National University of Science and Technology, Islamabad, PAK

Corresponding author: Abdul R. Azeem, darazeem7@gmail.com

\section{Abstract \\ Purpose}

Antimicrobial resistance (AMR) has become a worldwide environmental and public health problem, causing more than 250,000 deaths per year. Unregulated usage, unsafe hospital practices, and misuse in veterinary contribute to the development of multidrug resistance in various bacteria. Hospital water was hypothesized to be a hotspot for AMR transmission because of (1) increased exposure to antibiotic load, (2) poor drainage and sanitation system, (3) interaction between environmental and clinical microbes. The purpose of the research was to assess the biodiversity and AMR in hospital tap waters.

\section{Methodology}

In this study, the microflora of the hospital tap water and hospital surfaces was observed by obtaining water samples from the intensive care unit (ICU), surgical wards, and washrooms. These were processed through membrane filtration and spread on seven different media (Aeromonas Medium, Azide Dextrose Agar, MacConkey Agar, Mannitol Salt Agar, Pseudomonas Cetrimide Agar, Salmonella Shigella Agar, and Thiosulfate Citrate Bile Salts Sucrose Agar). Surface samples were collected from the faucet, basin, and drain and directly spread on the media plates. Isolates were identified using standard bacteriological and biochemical tests.

Kirby-Bauer disk diffusion method was performed using 21 antibiotic disks from 10 different antibiotic classes. They included ampicillin (AMP), amoxicillin (AML), piperacillin-tazobactam (TZP), cefipime (FEP), cefoxitin (FOX), ceftazidime (CAZ), ceftriaxone (CRO), imipenem (IMP), meropenem (MEM), ciprofloxacin (CIP), moxifloxacin (MXF), levofloxacin (LEV), amikacin (AK), gentamicin (CN), tigecycline (TGC), aztreonam (ATM), erythromycin (E), clindamycin (DA), rifampicin (RD), colistin (CT), and chloramphenicol (C). The results were interpreted according to EUCAST guidelines for the antibiogram of the isolates; 38 isolates were selected out of 162 based on different parameters for genotyping and detection of six betalactamase genes (blaSHV, blaTEM, blaCTX-M, blaOXA, blaKPC, blaNDM).

\section{Results}

Among these 162 isolates, 82 were obtained from water sources and 80 were collected from surfaces (faucet, basin, drain). The isolates included a variety of bacteria including Aeromonas spp. (20\%), Klebsiella spp. (13\%), Staphylococcus aureus (13\%), Pseudomonas spp.(10\%), Escherichia coli (9\%), Vibrio spp. (8\%), Enterococcus spp. (6\%), Shigella spp. (6\%), Salmonella spp. (4\%), Acinetobacter spp. (3\%), Staphylococcus epidermitis (3\%), Streptococci spp. (2\%), Proteus spp. (1\%), Citrobacter spp. (1\%), and Serratia spp. (1\%). A diverse range of microbes were identified including clinically relevant bacteria, which shows that the urban water cycle is already contaminated with multidrug-resistant microflora of the hospital settings. Macrolide and lincosamide showed the highest resistance followed by penicillin, monobactam, and cephalosporins. blaSHV and blaTEM were prevalent in samples. blaNDM was also found which manifests as a real threat since it causes resistance against carbapenems and colistin, antibiotics reserved as a last resort against infections.

\section{Conclusions}

This study presented the ground reality of antibiotic resistance in Pakistan and how its subsequent spread poses a great threat to the strides made in the field of medicine and public health. Strict regulations regarding antibiotic usage, hospital effluent, and urban water sanitation must be imposed to curb the devastating effects of this increasing phenomenon.

Categories: Infectious Disease, Public Health, Environmental Health

Keywords: colistin resistance, multi-drug resistant bacteria, beta-lactamases, hospital surfaces, hospital tap water, antibiotic resistance genes, transmission, antimicrobial resistance

\section{Introduction}


Antimicrobial resistance (AMR) has become a worldwide environmental and public health issue resulting in more than 700,000 deaths per year [1]. Water is considered to be the most important vehicle for the dissemination of antibiotic resistance in the environment due to its interaction in every compartment and its linkage with the human-associated microbiota. This is especially true for low-income countries where poor hygiene and sanitation practices further aggravate the problem [2]. The major intersection follows two routes, which come together with a full circle. The first one being the discharge of resistant bacteria in the environment through different sources and the second one being the presence of such bacteria in our urban water system which is consumed by the public.

The most significant amount of antibiotic residues and resistant bacteria are injected into the aquatic ecosystem through hospital water [3]. Hospitals in Pakistan have been estimated to produce 25,000 tons of waste each year containing about $1.4 \mu \mathrm{g} / \mathrm{L}$ to $236.6 \mu \mathrm{g} / \mathrm{L}$ of antibiotic residues, which are left untreated and added to surface water as such [4]. Untreated hospital effluents, entering into the municipal sewage pose a greater threat to the community where mixing of sewage water with drinking water is a common phenomenon [5].

This water ultimately makes its way back as contaminated tap water in hospitals and exposes the already ill and immunocompromised patients to several bacteria through ingestion [6]. The water used in hospital washbasins and washrooms has been claimed to have caused many nosocomial infections due to easy transmission channels between points of contact where patients are exposed to water while bathing, washing their hands, exposure to medical equipment, and through health workers and medical personnel [7]. The presence of antibiotic resistance bacteria (ARB) in such settings increases the chance of genetic transmission between microbes and results in increasing the load of antibiotic resistance and probable evolution into multidrug resistance bacteria [8].

The presence of antibiotic residues, pollutants, and nutrients in the wastewater serves as a selective pressure for the microbes to develop resistance and spread it via mutation or horizontal gene transfer [9]. Among many genetic determinants, resistance in Gram-negative microbes is mostly attributed to extendedspectrum $\beta$-lactamases (ESBL). ESBL enzymes have the capability to hydrolyze almost all beta-lactams which hinders the first-line defense against many infections. In recent times, blaCTX-M has become the most prevalent ESBL and together with blaSHV and blaTEM contribute to resistance against penicillins, oxyimino-cephalosporins, and monobactams [10]. Carbapenems and Cephamycins were the next drugs of choice against ESBL bacteria but the prevalence of blaOXA, blaKPC, and recently discovered blaNDM have also rendered these ineffective against resistant organisms.

To date, no study had been done in Pakistan on bacteria present in hospital water and their antibiotic resistance patterns. Keeping this in view, this study was designed to discern the prevailing issue of increased antibiotic resistance and antibiotic-resistant bacteria in the hospital water and surfaces with the aim to study their genes and consequent prevalence to better understand the dissemination of these organisms in the water and hospital environment.

\section{Materials And Methods Sample collection}

Three governmental tertiary care hospitals located in different cities were targeted for the sample collection from both water and surface sources to include and observe a range of microbiota. Intensive care units (ICU), surgical wards, and washrooms were selected to check the prevalence of bacteria and antibiotic resistance. The water samples were collected in a $50 \mathrm{ml}$ falcon tube after letting the tap run for two to three minutes to flush out the cold water. The tubes were then, sealed with the film to prevent contamination.

Swab samples were obtained through sterile swab sticks at three different sites: faucet, basin, and drain to study the bacterial inflow from the water system, the bacterial retention at sinks, and ultimate outflow to sewerage through the drainage system. After scrubbing on the respective surfaces, swabs were suspended in the transport medium and capped tightly to prevent contamination. All the samples were put in the ice container and processed within six hours of sampling with Karachi samples taking a few hours more. The experiments were performed in duplicates.

\section{Sample processing}

The water was passed through the membrane filter of $0.45 \mu \mathrm{m}$ and then placed gently on the nutrient medium with the sterile forceps. This was incubated at $37^{\circ} \mathrm{C}$ for 24 hours for the enrichment of microbes. The growth was observed the next day and the bacterial suspension was made in $1 \mathrm{ml}$ sterile saline solution. This was then, spread onto seven different media (Aeromonas Medium, Azide Dextrose Agar, MacConkey Agar, Mannitol Salt Agar, Pseudomonas Cetrimide Agar, Salmonella Shigella Agar, and Thiosulfate Citrate Bile Salts Sucrose Agar) to allow the growth of a multitude of microbes according to the nutritional specifications and conditions. Likewise, the surface samples were directly spread on the mentioned media on the first day of sampling and incubated at $37^{\circ} \mathrm{C}$ for 24 hours. Different colonies were picked and identified using standard bacteriological analysis protocols and biochemical tests.

\section{Antibiotic susceptibility assay}


This assay was performed using the standard Kirby-Bauer disk diffusion method on Mueller Hinton Agar (MHA) with EUCAST guidelines for the antibiogram of the isolates. The inoculum was prepared by suspending 24 hour fresh colonies in $1 \mathrm{ml}$ of sterile saline solution equating the turbidity to that of 0.5 McFarland and swabbed on the media plate. Twenty-one antibiotics disks were dispensed on the surface namely including ampicillin (AMP), amoxicillin (AML), piperacillin-tazobactam (TZP), cefipime (FEP), cefoxitin (FOX), ceftazidime (CAZ), ceftriaxone (CRO), imipenem (IMP), meropenem (MEM), ciprofloxacin (CIP), moxifloxacin (MXF), levofloxacin (LEV), amikacin (AK), gentamicin (CN), tigecycline (TGC), aztreonam (ATM), erythromycin (E), clindamycin (DA), rifampicin (RD), colistin (CT), and chloramphenicol (C). These agents were chosen on the basis of their importance in treating bacterial infections and for diverse representation of different antimicrobial classes. The plate was incubated at $37^{\circ} \mathrm{C}$ for $18-24$ hours and examined. The diameters of the zone of inhibition were measured against each antibiotic including the diameter of the disk.

\section{Isolation of genomic DNA}

Genomic DNA was extracted using the salting-out method where the fresh overnight liquid cultures are used for the isolation of DNA. Of the 162 isolates, only 38 (Table 1 ) were chosen for genotyping based on the following criteria: the geographical distribution, the varied sources, and higher antibiotic resistance.

\begin{tabular}{|c|c|c|c|c|c|c|c|c|}
\hline \multicolumn{3}{|c|}{ Selected AMR isolates (RWP) } & \multicolumn{3}{|c|}{ Selected AMR isolates (LH) } & \multicolumn{3}{|c|}{ Selected AMR isolates $(\mathrm{KH})$} \\
\hline Isolate & Source & Probable isolate & Isolate & Source & Probable Isolate & Isolate & Source & Probable Isolate \\
\hline R1K1 & ICU & Klebsiella spp & L2K1 & ICU & Escherichia coli & K3A1 & ICU & Aeromonas spp \\
\hline R2K1 & ICU & Salmonella spp & L1S2 & ICU & Shigella spp & K2K1 & ICU & Escherichia coli \\
\hline R3K2 & Ward & Escherichia coli & L4M1 & Ward & Staphylococcus aureus & K4K1 & Ward & Escherichia coli \\
\hline R4M1 & Ward & Staphylococcus epidermidis & L3A1 & Ward & Aeromonas spp & K5A1 & Ward & Aeromonas spp \\
\hline R5A1 & Washroom & Aeromonas spp & L5K1 & Washroom & Escherichia coli & K7K1 & Washroom & Acinetobacter spp \\
\hline R6K1 & Washroom & Klebsiella spp & L5A1 & Washroom & Aeromonas spp & K7S1 & Washroom & Shigella spp \\
\hline A11 & R Faucet & Aeromonas spp & K42 & L Faucet & Salmonella spp & K101 & K Faucet & Acinetobacter spp \\
\hline K72 & R Faucet & Klebsiella spp & T41 & L Faucet & Vibrio spp & S101 & K Faucet & Shigella spp \\
\hline $\mathrm{S} 21$ & R Basin & Escherichia coli & A52 & L Basin & Aeromonas spp & P111 & K Basin & Pseudomonas spp \\
\hline A22 & R Basin & Aeromonas spp & K51 & L Basin & Escherichia coli & D111 & K Basin & Enterococcus spp \\
\hline K32 & R Drain & Escherichia coli & $\mathrm{S} 62$ & L Drain & Salmonella spp & D121 & K Drain & Enterococcus spp \\
\hline \multirow[t]{3}{*}{ A31 } & R Drain & Aeromonas spp & A61 & L Drain & Aeromonas spp & S121 & K Drain & Escherichia coli \\
\hline & & & & & & K1S1 & Sea & Klebsiella spp \\
\hline & & & & & & K1P1 & Sea & Pseudomonas spp \\
\hline $\begin{array}{l}\text { TABL } \\
\text { distri }\end{array}$ & 1. Sel & $\begin{array}{l}\text { Ion of Isolates: } 38 \\
\text { ir source, and the }\end{array}$ & sis & ce pa & $\begin{array}{l}\text { lected Keeping } \\
\text { ern. }\end{array}$ & IIII & eII & apnic \\
\hline
\end{tabular}

Liquid culture of $1.5 \mathrm{ml}$ was centrifuged at maximum speed for one minute to achieve the pellet cells. This was repeated two to three times to get a thick pellet. The pellet was suspended in $600 \mu \mathrm{l}$ lysis buffer by soft pipetting after removing the supernatant. This mixture was incubated at $37^{\circ} \mathrm{C}$ for one hour.

Six hundred microliter of $5 \mathrm{M} \mathrm{NaCl}$ was then added to the mixture for protein precipitation. This was vortexed slowly for 15 seconds before putting it in a centrifuge at 10,000 RPM for 10 minutes. Following the step, the upper aqueous layer was carefully transferred to a new tube. This step was repeated until the white protein layer completely disappeared.

For the precipitation of DNA, 2.5 or 3 volumes of absolute chilled ethanol were added to the separated aqueous layer and gently mixed. This was refrigerated at $-20^{\circ} \mathrm{C}$ for 30 minutes followed by centrifugation at maximum speed with $4{ }^{\circ} \mathrm{C}$ temperature for 15 minutes. After discarding the supernatant, the DNA was washed with $1 \mathrm{ml}$ of chilled $70 \%$ ethanol, which was centrifuged at the same conditions for two minutes. The supernatant was discarded and the DNA pellet was air-dried at room temperature by inverting the tube on a paper towel. The DNA was then suspended in $600 \mu \mathrm{l}$ TE Buffer after drying. 


\section{Cureus}

\section{PCR for detection of antibiotic-resistance genes}

Six $\beta$ lactam genes were subject to be detected in the selected isolates namely: blaSHV, blaTEM, blaCTX-M, blaNDM, blaOXA, and blaKPC. The primer sequence and amplicon size are given in Table 2. Each PCR reaction consisted of $1 \times$ reaction buffer, $1.2 \mu \mathrm{l}$ of $50 \mathrm{mM} \mathrm{MgCl}_{2}, 0.5 \mu \mathrm{l}$ of $0.2 \mathrm{mM}$ dNTPs, $2 \mu \mathrm{l}$ of $1 \mathrm{mM}$ of each primer, $0.2 \mu \mathrm{l}$ Taq DNA polymerase, and $2 \mu \mathrm{l}$ of target DNA (final reaction volume of $25 \mu \mathrm{l}$ ). PCR products were electrophoresed through $2 \%$ agarose and ethidium bromide staining.

\begin{tabular}{|c|c|c|}
\hline Primer & Primer sequence $\left(5^{\prime}-3^{\prime}\right)$ & Amplicon size \\
\hline blaSHVF & CGCCATTACCATGAGCGATA & \multirow{2}{*}{86} \\
\hline blaSHVR & CGCAAAAAGGCAGTCAATCC & \\
\hline blaTEMF & AAGTTGCAGGACCACTTCTG & \multirow{2}{*}{202} \\
\hline blaTEMR & GCACCTATCTCAGCGATCTG & \\
\hline blaCTX-MF & CGATGTGCAGTACCAGTAA & \multirow{2}{*}{585} \\
\hline blaCTX-MR & TTAGTGACCAGAATCAGCGG & \\
\hline blaNDMF & GGTTTGGCGATCTGGTTTTC & \multirow{2}{*}{621} \\
\hline blaNDMR & CGGAATGGCTCATCACGATC & \\
\hline blaOXAF & GCGTGGTTAAGGATGAACAC & \multirow{2}{*}{438} \\
\hline blaOXAR & CATCAAGTTCAACCCAACCG & \\
\hline blaKPCF & CGTCTAGTTCTGCTGTCTTG & \multirow{2}{*}{798} \\
\hline blaKPCR & CTTGTCATCCTTGTTAGGCG & \\
\hline
\end{tabular}

TABLE 2: Amplicon size of detected genes: Six $\beta$ lactam genes were subject to be detected in the selected isolates namely: SHV, TEM, CTXM, NDM, OXA, and KPC.

The primer sequences and amplicon size is given in the table.

\section{Results}

\section{Bacterial diversity}

A total of 162 isolates were obtained from three major cities of Pakistan Rawalpindi (71), Lahore (57), and Karachi (34) as shown in Figure 1. Among these 162 isolates, 82 were obtained from water sources and 80 were collected from surfaces (faucet, basin, and drain). The isolates included a variety of bacteria including Aeromonas spp. (20\%), Klebsiella spp. (13\%), S. aureus (13\%), Pseudomonas spp. (10\%), E. coli (9\%), Vibrio spp. (8\%), Enterococcus spp. (6\%), Shigella spp. (6\%), Salmonella spp. (4\%), Acinetobacter spp. (3\%), $S$. epidermitis (3\%), Streptococci spp. (2\%), Proteus spp. (1\%), Citrobacter spp. (1\%), and Serratia spp. (1\%). Among the three surface sources checked, the drain was the most contaminated which suggests that the healthcare workers and patients who wash their hands in the basin are involved in spreading clinical bacteria into the drainage, which ultimately leads to sewage lines. The faucet was the least contaminated source found (Figure 2). 


\section{Cureus}

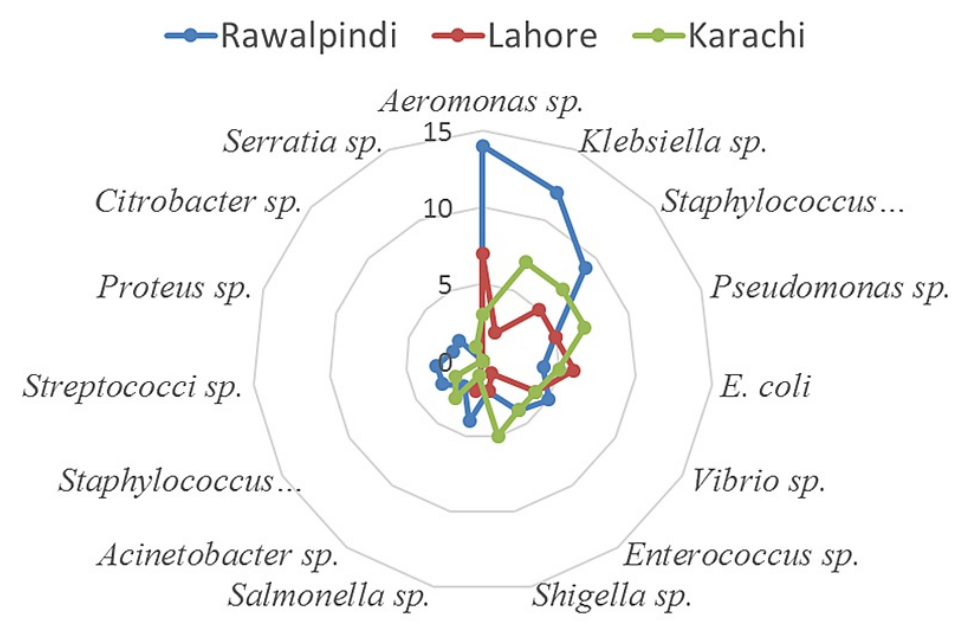

FIGURE 1: Illustrates the variety of species isolated from three different cities of Pakistan.

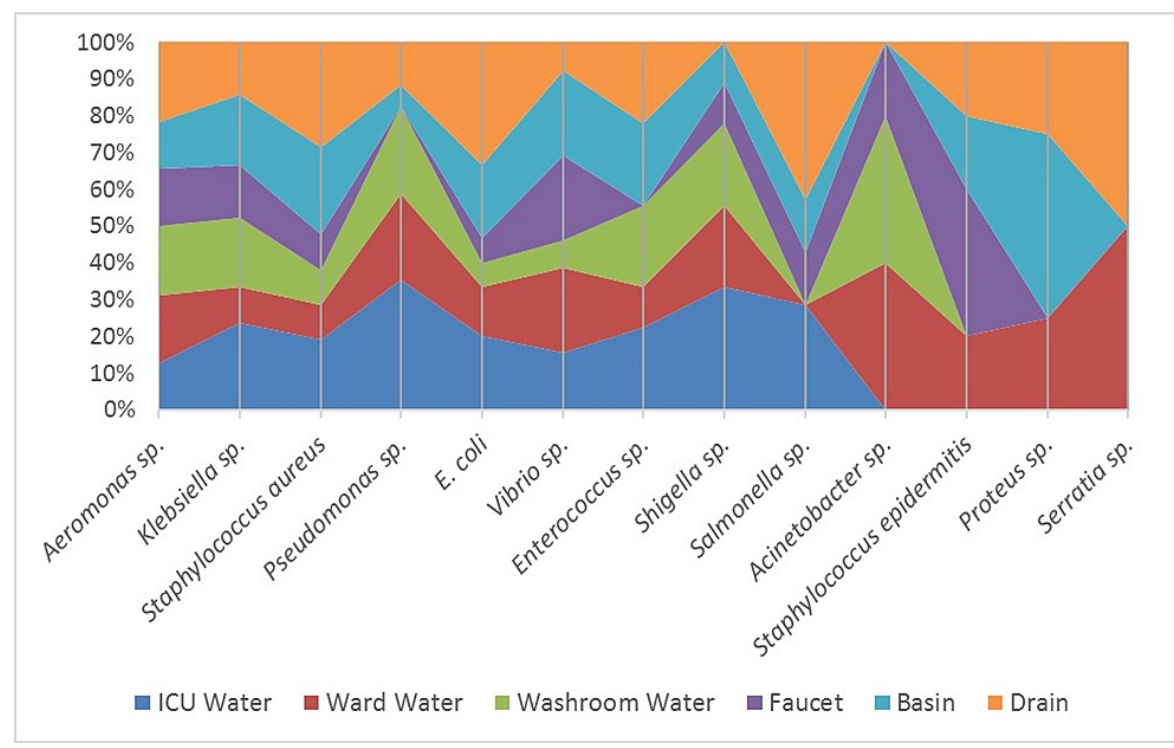

FIGURE 2: Illustrates the variety of species isolated from three selected water and surface sources each.

\section{Antibiotic susceptibility}

Among the 10 different classes of antibiotics tested, most resistance against macrolides was observed while resistance against fluoroquinolones was the least with levofloxacin being the most effective in this class as shown in Figure 3. Overall, colistin was found to be the most effective antibiotic with $52(32 \%)$ resistance among 162 isolates. Out of the total 162 isolates, about 118 (73\%) were extensively drug-resistant (XDR), 28 (17\%) were multidrug-resistant (MDR) while only 16 (10\%) were susceptible. Among Rawalpindi water isolates, tap water from washrooms contained the maximum number of XDR bacteria followed by tap water from ICUs. 


\section{Cureus}

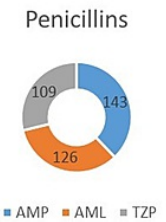

Aminoglycosides

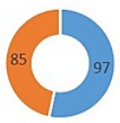

- $\mathrm{AK}$ = $\mathrm{CN}$

Lincosamide

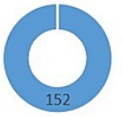

$\because \mathrm{DA}$
Cephalosporins

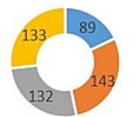

\# FEP $=$ FOX $\approx$ CAZ $=$ CRO

Tetracycline

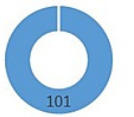

Miscellaneous

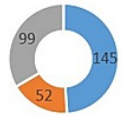

$\approx \mathrm{RD} \approx \mathrm{CT} \| \mathrm{C}$
Carbapenems

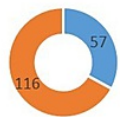

$\approx$ IPM $=$ MEM

Monobactam

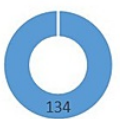

$=$ ATM
Flouroquinolones

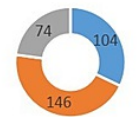

= CIP $=$ MXF $=$ LEV

Macrolide

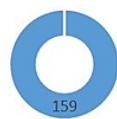

- $\mathrm{E}$

FIGURE 3: Illustrates the antibiotic resistance of 162 water and surface isolates.

Ampicillin (AMP), amoxicillin (AML), piperacillin-tazobactam (TZP), cefipime (FEP), cefoxitin (FOX), ceftazidime (CAZ), ceftriaxone (CRO), imipenem (IMP), meropenem (MEM), ciprofloxacin (CIP), moxifloxacin (MXF), levofloxacin (LEV), amikacin (AK), gentamicin (CN), tigecycline (TGC), aztreonam (ATM), erythromycin (E), clindamycin (DA), rifampicin (RD), colistin (CT), and chloramphenicol (C).

Among the surface isolates, drug resistance was more profound in Rawalpindi drain isolates, in which $23 \%$ were resistant to all the drugs tested while $92 \%$ of the basin surface and $75 \%$ of faucet surface isolates were also found to be XDR. Isolates from Lahore had 100\% XDR bacteria in ICU tap water and washroom tap waters. Among the Lahore faucet, basin, and drain surface isolates, XDR bacteria were found to be $100 \%$, $67 \%$, and $83 \%$, respectively. Among the tap water samples collected from Karachi, ICU water samples showed 60\% XDR bacteria while ward water isolates showed 67\% XDR bacteria. Among the faucet, basin, and drain surface isolates, XDR were tested to be $80 \%, 67 \%$, and $77 \%$, respectively (Figure 4 ).

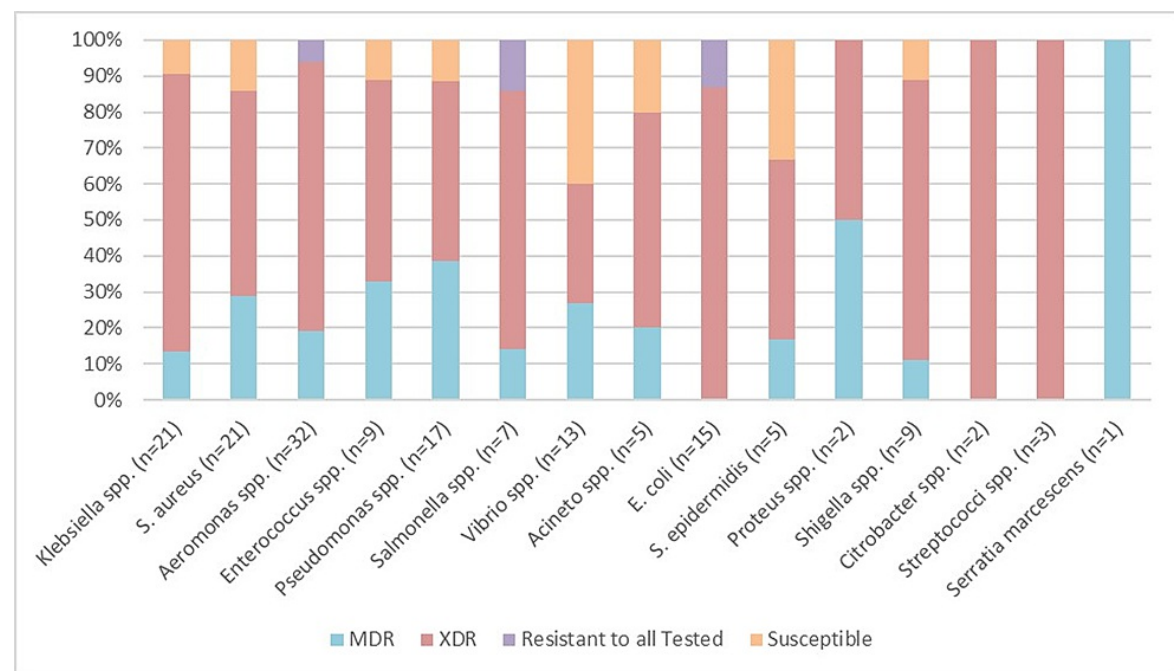

FIGURE 4: Illustrates the antibiotic resistance in all isolates of bacteria.

All isolates of Streptococci and Citrobacter were tested to be XDR. Klebsiella spp. were found to have 81\% XDR isolates and 14\% MDR isolates as shown in Figure 4. Similarly, S. aureus also had a higher ratio of 1.8 XDR to MDR bacteria. E. coli, another common bacteria showed $13 \%$ of isolates that were resistant to all the antibiotics tested while the remaining $87 \%$ of its isolates were found to be XDR. Salmonella spp. showed $14 \%$ isolates that were resistant to all the antibiotics tested. Enterococcus spp. also had $56 \%$ of the isolates classified as XDR. Aeromonas, an environmental bacteria and a fish pathogen, had $6 \%$ isolates that were resistant to all the antibiotics tested, which is a matter of great concern. 


\section{Cureus}

\section{Prevalence of beta-lactam genes}

The isolates showed a varied presence of the six beta-lactam genes, the most prevalent being blaTEM(68\%) with blaSHV (66\%) following closely behind. blaCTX-M was found in $29 \%$ with $18 \%$ blaNDM and $16 \%$ blaOXA. There was no blaKPC gene detected. The overall prevalence of genes was detected to be higher in Lahore isolates. The Karachi isolates showed no detection of the blaCTX-M gene, however, it had the highest frequency of blaSHV (26\%). Rawalpindi isolates showed the highest frequency of blaSHV (16\%) and blaCTX$M(16 \%)$. Among the species, Aeromonas spp. and E. coli were found to possess all five genes that were detected while Acinetobacter spp., Salmonella spp., and Klebsiella spp. also harbored four out of five genes. The highest prevalence of blaNDM was found in Acinetobacter spp. (Figures 5-6).

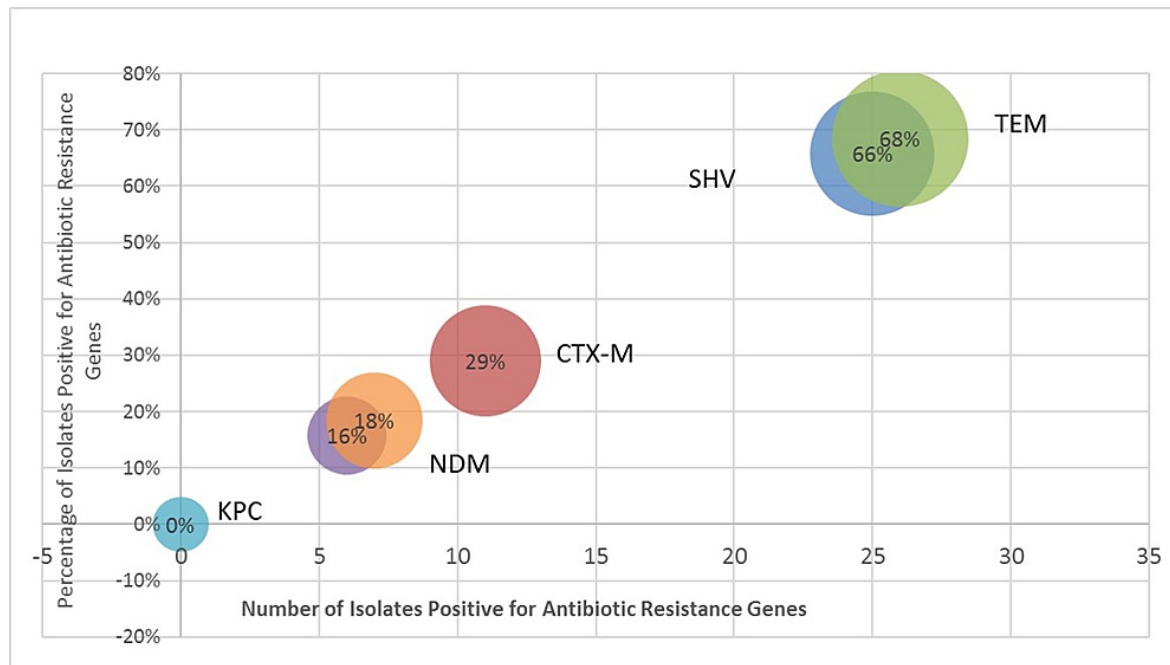

FIGURE 5: Illustrates the number of genes detected in selected isolates.

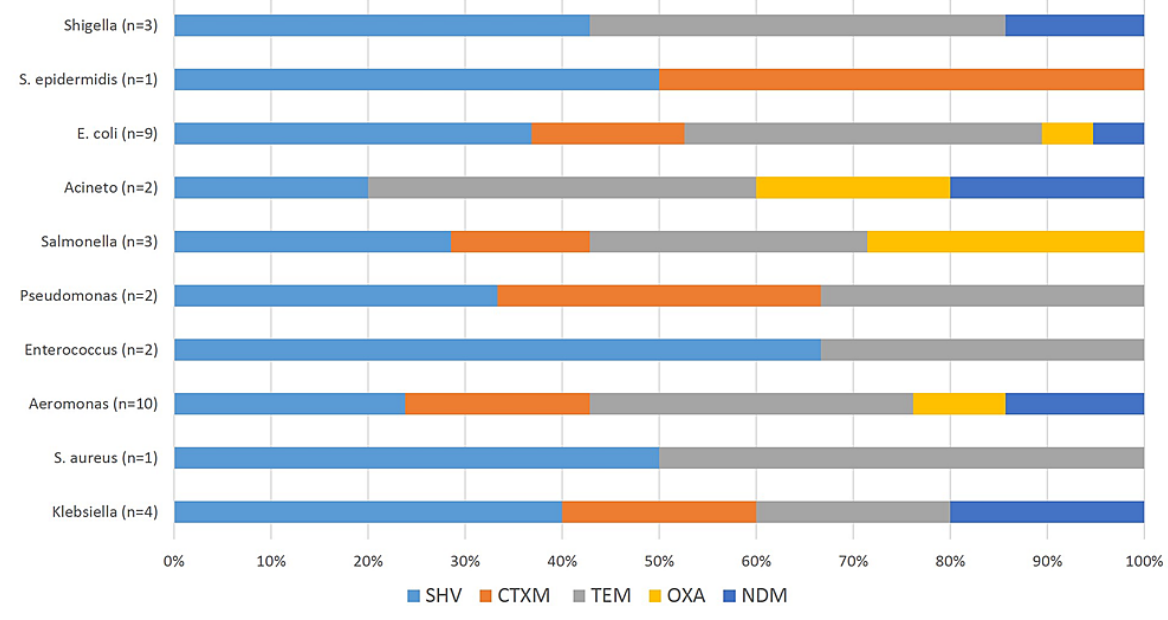

FIGURE 6: Illustrates the antibiotic resistance genes in bacterial isolates.

\section{Discussion}

The hospital environment is an intricate ecosystem that has usually been overlooked as a potential reservoir for bacteria but with the rate of nosocomial infections on the rise; this has become a critical area for the study of microbes. The transmission of pathogens via surface contamination, lack of proper handwashing practices among health care workers, and the water system increased the incidence rate of hospital-acquired infections among the patients [11].

Furthermore, the increase in the antibiotic resistance patterns of the microbes has forced the scientific community to look into the source of such pathogens and their mechanisms of acquired resistance in different environmental compartments. Water bodies were also considered to be a reservoir for the antibiotic resistance, especially because it facilitates the interaction of pathogenic bacteria with the non-pathogenic 
In the present study, of 162 isolates, 127 were Gram-negative while only 35 isolates were Gram-positive. Similar ratios were found in other studies that observed the bacterial diversity in hospital settings with the prevalence of Gram-negative bacteria [13]. In a study conducted on river waters, $81.8 \%$ of Aeromonas samples were found to be multidrug-resistant to the most commonly used antibiotics [14]. The results are in coherence with our study where $100 \%$ resistance was shown towards ampicillin and more than $80 \%$ towards amoxicillin, aminoglycosides, and macrolides. Cephalosporins, which were considered to be suitable drugs [15] against Aeromonas also showed more than $80 \%$ resistance to ceftriaxone whereas susceptibility to ciprofloxacin has decreased to $44 \%$, concurrent with the study [16].

Similarly, Klebsiella species has also shown an increasing trend of resistance against commonly used antibiotics with cefotaxime resistance increased from $75 \%$ to $94 \%$, ciprofloxacin went from $64 \%$ to $84 \%$, and carbapenems from $2.4 \%$ to $52 \%$ [17]. The trend in our study is on the higher end of the spectrum with $90 \%$ resistance to ciprofloxacin, cefoxitin, and erythromycin, $80 \%$ towards ceftriaxone, and $40 \%$ towards carbapenems. Carbapenem resistance is owed to recently discovered, blaNDM genes and blaCTXM, both of which were present in our isolates. This leaves colistin as the only suitable option with $70 \%$ susceptibility in Klebsiella isolates coherent with a study done in Europe [18].

Pseudomonas species was the third most common organism isolated in our study with $90 \%$ isolates as multidrug-resistant. More than $80 \%$ resistance was shown towards cephalosporins, penicillins, and macrolides coherent with other studies [19]. The least resistance was observed against ciprofloxacin (30\%) and colistin (10\%) supported by a study done in Karachi [20]. This pattern of resistance is mainly due to the expanded beta-lactam activity of these strains which is contributed to beta-lactamase genes. Our tested isolates were shown to carry extended-spectrum beta-lactamase genes blaTEM, blaSHV, and blaCTXM. The frequencies of blaTEM and blaSHV genes in the bacterial isolates were calculated to be $46 \%$ and $34 \%$, respectively [21], in another study which is similar to our findings.

A study conducted on water samples from hospital sources found $S$. aureus to be the most predominant organism. Comparatively, it constituted only $13 \%$ of total isolates in our study. Different studies have shown Staphylococcus to be multidrug-resistant with $100 \%$ resistance against amoxicillin, streptomycin, ceftriaxone, and erythromycin, $83 \%$ to gentamycin and $78 \%$ to cefoxitin and ciprofloxacin [21]. These findings were supported by our study as more than $80 \%$ of resistance was shown towards these drugs classes. A shifting trend was observed in our study from a study that reported amikacin and levofloxacin as a susceptible antibiotic against Staphylococcal isolates where resistance has increased to $58 \%$ and $52 \%$, respectively, with $96 \%$ sensitivity to imipenem as reported in a study from Peshawar [21]. Staphylococcus epidermidis also exhibited resistance to macrolides, cephalosporins, and fluoroquinolones as reported in other studies [22]. Researchers have associated multidrug resistance with blaTEM genes along with blaCTXM and blaSHV that were prevalent in our Staphylococcal isolates.

Organisms like E. coli, Vibrio, Enterococci, and Shigella have been known to cause water-borne diseases like cholera and dysentery. Combined, they made $23 \%$ of the total isolates. Similar resistance patterns were observed in isolates of $E$. coli with $100 \%$ resistance towards cephalosporins, more than $90 \%$ resistance against ampicillin, more than $80 \%$ resistance towards macrolides, and $68 \%$ against fluoroquinolones. This was also supported by a study that reported $100 \%$ resistance towards penicillins, more than $80 \%$ towards first and second-generation cephalosporins, and 20\% towards fluoroquinolones and macrolides [23]. On contrary, Vibrio species showed $90 \%$ susceptibility to fluoroquinolones and $80 \%$ susceptibility to carbapenems consistent with findings in China [24] but resistance patterns were similar against penicillins, erythromycin, and clindamycin as in other isolates in our study. The resistance in both Enterococci and Shigella was $100 \%$ towards erythromycin and ceftriaxone, followed by $90 \%$ against ampicillin and cefoxitin concurrent with other studies [25]. Resistance against ceftriaxone (20\%) and ciprofloxacin (12\%) in Shigella has risen in the past seven years to $68 \%$ and $58 \%$, respectively, in our study compared to the study done in Faisalabad. This overall resistance against all these drugs has been conferred to the bacteria's innate resistance [26]. blaSHV and blaTEM have been found to confer resistance against many antibiotics and have been the most prevalent in E. coli, Vibrio, Enterococci, and Shigella. E. coli had the maximum number of resistance genes including blaCTXM, blaOXA, and blaNDM.

XDR typhi is an extensively drug-resistant strain of Salmonella typhi and is resistant to all the antibiotics recommended for typhoid fever except azithromycin and carbapenems. The isolates in our study have exhibited $100 \%$ resistance towards ampicillin, ciprofloxacin, aztreonam, clindamycin, and colistin with similar results reported in other studies [27]. Salmonella isolates were found to possess blaSHV, blaTEM, blaCTXM, and blaOXA, all of which explain the resistance pattern shown by the isolates. ESBL pattern of resistance has also been observed in Acinetobacter and Pneumococci isolates with the highest resistance against penicillins, cephalosporins, and fluoroquinolones consistent with other findings [28]. Recently, several serotypes of $S$. pneumoniae have been isolated which show $100 \%$ resistance towards penicillins and cephalosporins with growing resistance towards macrolides and fluoroquinolones. Growing resistance against chloramphenicol has also been recently discovered where $36 \%$ resistance was reported against the drug [29], but it contradicts our findings of $100 \%$ sensitivity towards it. Proteus species on the other hand has shown 100\% resistance towards chloramphenicol as well as penicillins, cephalosporins (all three generations), and macrolides. It has shown susceptibility towards piperacillin-tazobactam [30] but our findings are contrary to these as $100 \%$ resistance was shown towards this drug with only susceptibility 


\section{Conclusions}

It was concluded in this study that Gram-negative bacteria have more suited features to be able to survive in the environment for longer periods of time, which is why they are of concern especially, in the hospital setting where the patients are more prone to catch an infection. This study suggests that hospital tap water habitat comprises a diverse range of microbes including the ones that have been identified as clinically relevant. The study also shows that the urban water cycle is already contaminated with the microflora of the hospital settings including Aeromonas, Klebsiella, Pseudomonas, Staphylococcus, and Vibrio species in abundance that are a threat to hospitalized patients, especially immunocompromised ones.

Most resistance was found against macrolides, lincosamides, monobactams, and penicillins. The most resistant pathogens were found to be in ICU compared to other wards because of more frequent antibiotic usage and the presence of immunocompromised patients, which call for stringent policies and infection control programs in the hospitals. Apart from this, many of the organisms in this study were found to be extended-spectrum beta-lactam (ESBL) producers. This manifests as a real threat, especially with blaNDM prevalence on the rise, which might result in resistance against carbapenems and colistin, antibiotics reserved as a last line of defense against infections. Public health measures for clean tap water, clean water supply in hospitals along with information sharing and stimulation of research in this field shall contribute towards bridging gaps and a better understanding of this increasing phenomenon. We need more research in this area involving more hospitals and medical setups around the country.

\section{Additional Information \\ Disclosures}

Human subjects: All authors have confirmed that this study did not involve human participants or tissue. Animal subjects: All authors have confirmed that this study did not involve animal subjects or tissue. Conflicts of interest: In compliance with the ICMJE uniform disclosure form, all authors declare the following: Payment/services info: All authors have declared that no financial support was received from any organization for the submitted work. Financial relationships: All authors have declared that they have no financial relationships at present or within the previous three years with any organizations that might have an interest in the submitted work. Other relationships: All authors have declared that there are no other relationships or activities that could appear to have influenced the submitted work.

\section{References}

1. Kalungia AC, Mwambula H, Munkombwe D, et al.: Antimicrobial stewardship knowledge and perception among physicians and pharmacists at leading tertiary teaching hospitals in Zambia: implications for future policy and practice. J Chemother. 2019, 31:378-87. 10.1080/1120009X.2019.1622293

2. Walker GT, Quan J, Higgins SG, et al.: Predicting antibiotic resistance in Gram-negative bacilli from resistance genes. Antimicrob Agents Chemother. 2019, 63: 10.1128/AAC.02462-18

3. Ahmad M, Khan AU: Global economic impact of antibiotic resistance: a review . J Glob Antimicrob Resist. 2019, 19:313-6. 10.1016/j.jgar.2019.05.024

4. Kumar R, Somrongthong R, Ahmed J: Effect of medical waste management trainings on behavior change among doctors versus nurses and paramedical staff in Pakistan [internet]. J Ayub Med Coll Abbottabad. 2016, 28:493-6.

5. Almakki A, Jumas-Bilak E, Marchandin H, Licznar-Fajardo P: Antibiotic resistance in urban runoff. Sci Total Environ. 2019, 667:64-76. 10.1016/j.scitotenv.2019.02.183

6. Ogawara $\mathrm{H}$ : Comparison of antibiotic resistance mechanisms in antibiotic-producing and pathogenic bacteria. Molecules. 2019, 24:3430. 10.3390/molecules24193430

7. Lerminiaux NA, Cameron AD: Horizontal transfer of antibiotic resistance genes in clinical environments . Can J Microbiol. 2019, 65:34-44. 10.1139/cjm-2018-0275

8. Wencewicz TA: Crossroads of antibiotic resistance and biosynthesis. J Mol Biol. 2019, 431:3370-99. 10.1016/j.jmb.2019.06.033

9. Ben Y, Fu C, Hu M, Liu L, Wong MH, Zheng C: Human health risk assessment of antibiotic resistance associated with antibiotic residues in the environment: A review. Environ Res. 2019, 169:483-93. 10.1016/j.envres.2018.11.040

10. Chokshi A, Sifri Z, Cennimo D, Horng H: Global contributors to antibiotic resistance. J Glob Infect Dis. 2019, 11:36-42. 10.4103/igid.jgid_110_18

11. Singh R, Singh AP, Kumar S, Giri BS, Kim KH: Antibiotic resistance in major rivers in the world: A systematic review on occurrence, emergence, and management strategies. J Clean Prod. 2019, 234:1484-505.

12. Umar M, Roddick F, Fan L: Moving from the traditional paradigm of pathogen inactivation to controlling antibiotic resistance in water - Role of ultraviolet irradiation. Sci Total Environ. 2019, 662:923-39. 10.1016/j.scitotenv.2019.01.289

13. Tajeddin E, Rashidan M, Razaghi M, et al.: The role of the intensive care unit environment and health-care workers in the transmission of bacteria associated with hospital acquired infections. J Infect Public Health. 2016, 9:13-23. 10.1016/j.jiph.2015.05.010

14. Harnisz M, Korzeniewska E: The prevalence of multidrug-resistant Aeromonas spp. in the municipal wastewater system and their dissemination in the environment. Sci Total Environ [Internet]. 2018, 20:37783.

15. Ahmed I, Rabbi MB, Sultana S: Antibiotic resistance in Bangladesh: a systematic review . Int J Infect Dis. 2019, 80:54-61. 10.1016/j.ijid.2018.12.017

16. Fernández-Bravo A, Figueras MJ: An update on the genus Aeromonas: taxonomy, epidemiology, and 
pathogenicity. Microorganisms. 2020, 8:129. 10.3390/microorganisms8010129

17. Martin RM, Bachman MA: Colonization, infection, and the accessory genome of Klebsiella pneumoniae . Front Cell Infect Microbiol. 2018, 8:4. 10.3389/fcimb.2018.00004

18. Subedi D, Vijay AK, Willcox M: Overview of mechanisms of antibiotic resistance in Pseudomonas aeruginosa: an ocular perspective. Clin Exp Optom. 2018, 101:162-71. 10.1111/cxo.12621

19. Ebrahimpour M, Nikokar I, Ghasemi Y, Sedigh Ebrahim-Saraie H, Araghian A, Farahbakhsh M, Ghassabi F: Antibiotic resistance and frequency of class 1 integrons among Pseudomonas aeruginosa isolates obtained from wastewaters of a burn center in Northern Iran. Ann Ig. 2018, 30:112-9. 10.7416/ai.2018.2202

20. Frequency and susceptibility pattern of Multidrug Resistant Pseudomonas aeruginosa in isolates of patients from a tertiary care hospital of Karachi, Pakistan [Internet]. (2019). Accessed: October 12, 2021: https://www.researchgate.net/publication/278730660_Frequency_and_susceptibility_pattern_of_Multidrug_Resistant_Pseudc

21. Okhonlaye O, Oluwatosin O: Occurrence of multidrug resistant Staphylococcus aureus and Klebsiella pneumoniae isolated from clinical and environmental samples in Ondo state, Nigeria. J Adv Microbiol [Internet. 2018, 5:1-10. 10.9734/JAMB/2018/40204

22. Lee JY, Monk IR, Gonçalves da Silva A, et al.: Global spread of three multidrug-resistant lineages of Staphylococcus epidermidis. Nat Microbiol. 2018, 3:1175-85. 10.1038/s41564-018-0230-7

23. Wang CY, Chen YH, Fang C, et al.: Antibiotic resistance profiles and multidrug resistance patterns of Streptococcus pneumoniae in pediatrics: a multicenter retrospective study in mainland China. Medicine (Baltimore). 2019, 98:e15942. 10.1097/MD.0000000000015942

24. Gladkikh AS, Feranchuk SI, Ponomareva AS, Bochalgin NO, Mironova LV: Antibiotic resistance in Vibrio cholerae El Tor strains isolated during cholera complications in Siberia and the Far East of Russia. Infect Genet Evol. 2020, 78:104096. 10.1016/j.meegid.2019.104096

25. Tian Y, Yu H, Wang Z: Distribution of acquired antibiotic resistance genes among Enterococcus spp. isolated from a hospital in Baotou, China. BMC Res Notes. 2019, 12:27. 10.1186/s13104-019-4064-Z

26. Okai M, Aoki H, Ishida M, Urano N: Antibiotic-resistance of fecal coliforms at the bottom of the Tama River, Tokyo. Biocontrol Sci. 2019, 24:173-8. 10.4265/bio.24.173

27. Ranjbar R, Farahani A: Shigella: antibiotic-resistance mechanisms and new horizons for treatment. Infect Drug Resist. 2019, 12:3137-67. 10.2147/IDR.S219755

28. Mechai A, Debabza M, Thabet R, Sedira H, Fadeleddine S, Mechai A: Occurrence and spread of betalactamases-producing enterobacteriaceae isolated from river receiving treated effluent of wastewater treatment plant. Desalin Water Treat. 2019, 147:156-63. 10.5004/dwt.2019.23763

29. Ajulo OH, Adetunji OV, Ajulo OM: Antibiotic sensitivity profiles of Salmonella typhimurium and E. coli O157: H7 isolates from ready to eat chicken meat in Ibadan-Nigeria. Asian Food Sci J. 2019, 10:1-17. 10.9734/afsi/2019/v10i130025

30. Rocha C, Bernal M, Canal E, et al.: First report of New Delhi metallo- $\beta$-lactamase carbapenemase-producing Acinetobacter baumannii in Peru. Am J Trop Med Hyg. 2019, 100:529-31. 10.4269/ajtmh.18-0802 